\title{
Using Phylogeny Approach on Ethnobotanical Bioprospecting for Leading Antimalarial Plant-Based Drug Discovery
}

\author{
Desy Liana $^{\mathrm{a}}$, Kanchana Rungsihirunrat ${ }^{\mathrm{a},{ }^{*}}$ \\ ${ }^{a}$ College of Public Health Sciences, Chulalongkorn University, Bangkok, 10330, Thailand \\ Corresponding author: *kanchana.r@chula.ac.th
}

\begin{abstract}
Ethnobotanical-directed bioprospecting has made a significant contribution to modern drug discoveries. However, merely relying on this approach may spend more expenditure, time-consuming, and lead exhaustive laboratory testing due to the tremendous data of medicinal plants used and the occurrence of placebo effect during traditional medical treatment. Combining the phylogeny approach with ethnobotanical bioprospecting may become new prospective tools to lead the plant-based drug discovery, including antimalaria. This study aimed to map the ethnomedicinal plants used by various indigenous cultures to investigate the clustered pattern of its antimalarial properties for future bioprospecting. The Internal Transcribed Spacer (ITS) region sequences of selected 280 medicinal plants taxa obtained from NCBI (National Center for Biotechnology Information) were aligned by MUSCLE multiple sequences alignments. They were further analyzed using the Maximum Likelihood Phylogenetic Test by MEGA X software to construct the phylogenetic tree. Our research revealed that the medicinal plant taxa for malaria treatment was clumped in several families, including Apocynaceae, Euphorbiaceae, Rubiaceae, Rutaceae, Fabaceae were strongly clumped along with plants used for fever in the Asteraceae family. Interestingly, our finding showed that these plants were clumped in the sub-family of antimalarial producing species, the Asteroidea. Furthermore, the strongly clumping pattern was also shown in the tribe Heliantheae alliance of this sub-family. This finding supports the predictive power of phylogeny for future bioprospecting to select the candidate taxa to lead the drug discovery.
\end{abstract}

Keywords - Phylogeny; bioprospecting; ethnomedicinal plants; malaria; drug discovery.

Manuscript received 18 Aug. 2020; revised 2 Feb. 2021; accepted 24 Feb. 2021. Date of publication 30 Apr. 2021. IJASEIT is licensed under a Creative Commons Attribution-Share Alike 4.0 International License.

\section{INTRODUCTION}

Plant material has been used from ancient times through traditional way utilization for the treatment of a various range of diseases. Different cultures in various regions may have their recipe to utilize the herbs as their primary health care [1]-[3]. Interestingly, these ethnobotanical data significantly contribute to drug discovery and development [4]-[6]. According to World Health Organization (WHO), 25\% of modern drugs are derived from plants [7], [8]. Before the emergence of the synthetic era, in the early $1900 \mathrm{~s}, 80 \%$ of medicines were obtained from the part of the plants [9], [10]. Bioactivity guide screening from ethnobotanical data becomes vogue to guide the modern drug discovery afterward [11], [12]. However, the tremendous numbers of ethnomedicinal plants from various cultures challenge making medicinal plants' target selection. Drug discovery via the traditional approach may require more expenditure and time, even struggling to keep pace with the rapid development of high throughput technologies [13], [14]. The effectiveness of this direct approach might be questioned because of many studies revealed that after testing in the laboratory, these ethnobotanical medicinal plants give less or none efficacy at all which may lead the time consumed research when only relying onto this approach [7], [15]. This might be happened because of the "placebo effect" during the treatment [16]. The patient might be healed by their own suggestion given from the traditional healer.

Phylogenetic approach may become new complementary tools which could be useful to predict the medicinal properties of the ethnobotanical plants. This based on the assumption on the chemicals-properties sharing possibility between related species [17]. This suggestion is supported by the study on a number of ethnomedicinal plants distributed in Nepal, South Africa Cap e and New Zealand which revealed that medicinal plants used to treat medical condition in the same therapeutic areas were showed concentrated in certain sites of the phylogenetic trees[7]. Furthermore, result from mapping the psychoactive plants using phylogenetic analysis also showed that the psychoactive properties were clumped on certain plant lineages [18]. 
Secondary metabolites are known to be the major compounds that contribute to the therapeutic effects. They are produced by specific gene clusters expressed continuously as a part of the defense mechanism; hence those chemicals might be evolutionarily conserved [19]-[21]. These compounds are generated by specific gene clusters which are expressed in a particular group of plants. Many commercial drug-based natural products have been investigated using phylogenetic tools and revealed that $67 \%$ of clinical trial drug and $80 \%$ approved drugs were concentrated in 30 and 17 drugproductive families. This finding suggested that drugs derived natural product is mostly generated from pre-existing prolificdrug families. The evolutionarily related species also showed more medicinal value than the isolated evolutionary species [22]. However, some studies showed that not all biochemicals are clustered phylogenetically (e.g., stimulant chemicals from many investigated psychoactive plants showed to be more scattered rather than hallucinogen and sedative chemicals) [23], which is lead the uncertain robustness using this approach hence require to be explored furtherly [19].

Malaria is a vector-borne infectious disease caused by Plasmodium parasites transmitted by Anopheles mosquitoes. In 2018, 405.000 death occurred from 228 million cases. Children in sub-Saharan Africa are the most threatened by this disease [24]. This disease still becomes a significant challenge in the public health sector and drug resistance. Artemisinin and derivatives, which is known as the core treatment, are reported to develop resistance and spread over in Southeast Asia [25]-[27]. Artemisinin resistance can be defined as the delay in parasite clearance, which may cause the clinical failure of treatment [28], [29]. Accordingly, people are to find the new candidate drug as a replacement or complement as an activity enhancer [30], [31].

Even though ethnobotanical bioprospecting has been made a significant lead to discover the commercial antimalarial drugs (the artemisinin and quinine), combining phylogenetic approach with the ethnobotanical bioprospecting may become a new promising strategy which able to narrow down the selection of the candidate plant for further investigation and could give a faster and efficient way to discover the plantbased drug. This research was the first attempt to map the medicinal plant, which is commonly used by various indigenous cultures worldwide to treat malaria and fever as its related symptom using phylogenetic tools to select the plants as antimalarial plant candidates.

\section{MATERIALS AND METHODS}

\section{A. Data collection}

Medicinal plant taxa list used by various indigenous cultures was obtained from literature search by reviewing published articles in ethnobotanical surveys and related literature that presented the plants' usage to treat malaria and fever as its related symptom. A plant list for another disease that has a different indication (medicinal plants for tuberculosis) was added to analyze on the broader sight whether the additional number of the samples may alter the clustered pattern of the phylogeny result. Literature databases including PubMed, Scopus, ScienceDirect, and Google
Scholar were used with the searching keyword: "ethnomedicine"/“ethnobotany", "traditional", "herbal medicine", "malaria"/"fever"/ "antipyretic"/"tuberculosis". Medicinal plant list database was created based on the literature search of ethnobotanical surveys conducted in various indigenous cultures in several tropical regions, including Africa (Uganda, Limpopo, Nigeria, Kenya, Ivory Coast, Ghana, Zimbabwe, Madagascar, and Senegal) and Indomalaya (Nepal, Iran, India, Bangladesh, Thailand and Indonesia).

Data extraction was performed with inclusion and exclusion criteria adapted and modified from Aid and Jeanmaire [23]:

- Only single used plants for treatment were included, medicinal plants in remedies were excluded

- Congeneric taxa were only presented once to avoid visually bias in the phylogenetic clustering due to frequent possibility giving the similar properties (e.g., Artemisia spp. represented several taxa of Artemisia annua, Artemisia afra, Artemisia brevifolia, and Artemisia gmelini).

Plant list database (The Plant List, http://www.theplantlist.org/), a working list of all plant species which is maintained by Royal Botanic Gardens, Kew and Missouri Botanical Garden, was used to verify the name and family of each taxa.

Internal Transcribed Spacer (ITS) region was used to construct a phylogenetic tree. The 605 species of medicinal plants taxa from 144 families were obtained from literature. However, only 280 plants taxa (280 genus from 105 family) were met the criteria, as well as the ITS sequences were available in National Center for Biotechnology Information (NCBI) database ( 77 plants taxa for malaria, 77 taxa for fever, 59 taxa for tuberculosis, and 67 multipurpose taxa). Hence this list was processed for further analysis.

\section{B. Phylogenetic Tree Construction and Clustered Pattern Analysis}

The sequences obtained from NCBI database were aligned using the default system in MUSCLE multiple sequence alignment while phylogenetic tree construction was performed using Maximum Likelihood Phylogenetic Test with bootstrapping 100 times MEGA X software. In addition, creating datasets, annotation, and made up of the interactive phylogenetic tree were done using Interactive Tree of Life (ITOL, https://itol.embl.de/) and Adobe Illustrator 2020. The clustered pattern of the plant's medical use was assessed descriptively by using Heatmap datasets in ITOL. Coding 0 (absent) and 1 (present) were used to indicate the presence of the plant's medical use. The workflow stage of the study was showed in Fig 1. The clustered pattern was determined descriptively by analyzing the clumping color on each clade branch for each medical therapeutic category. Red, blue, and green colors were applied in every clade, which showed the cluster signal for ethnomedicinal plants used for malaria, fever, and tuberculosis, respectively. The clustered pattern was further analyzed by identifying each taxa family, subfamilies, and tribes based on current classification. 


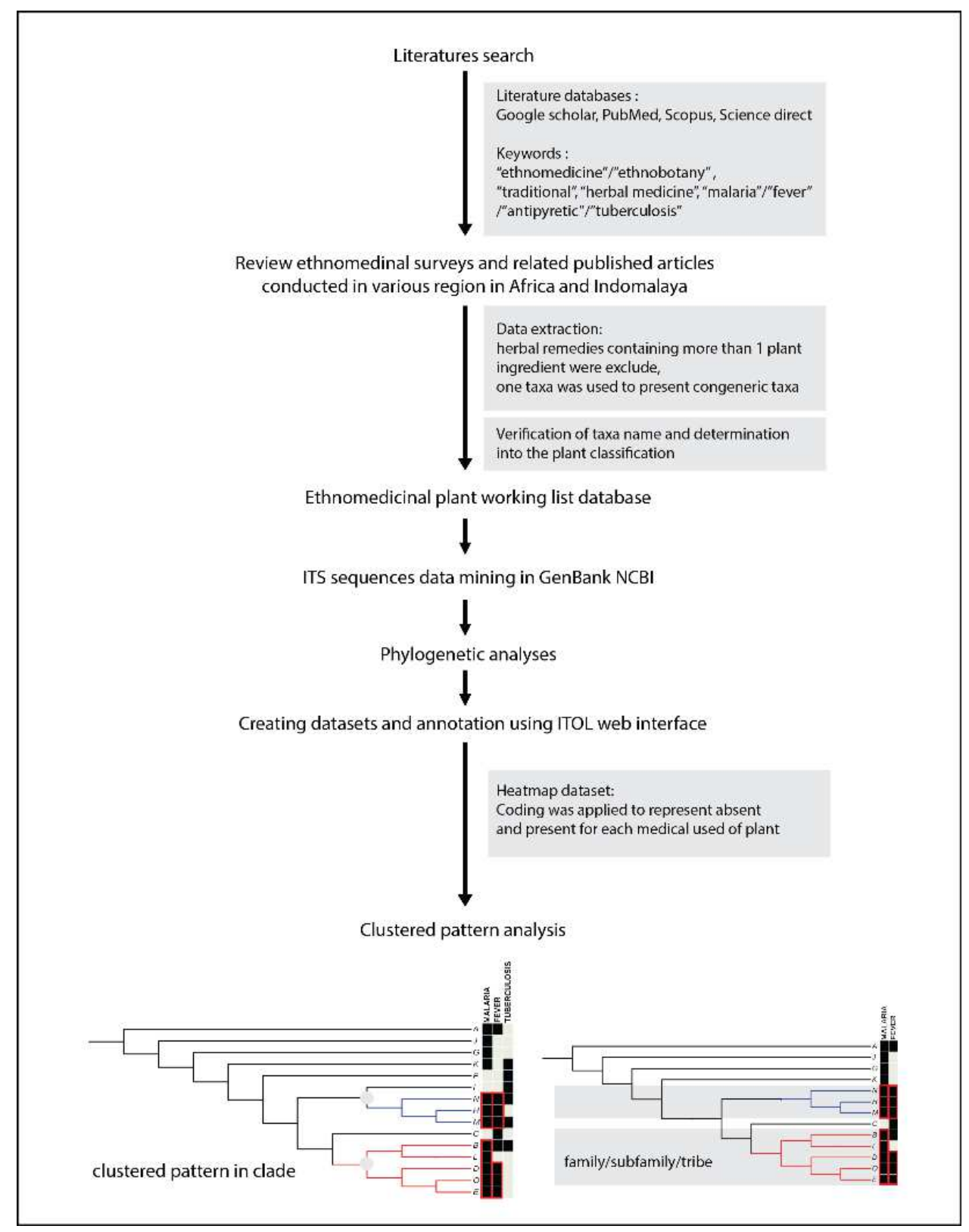

Fig. 1 Workflow stages of the study

\section{RESULTS AND DISCUSSION}

Effort in discovering new antimalarial drugs is being performed by various approaches but still face their own challenge, especially to find the systematic and efficient method to lead the drug discovery. The conventional approach using bioactivity guide screening from the ethnobotanical data has led to current commercial drug discoveries. However, this strategy is laborious, timeconsuming, and might spend more expenditure. The increase of molecular and non-molecular data-parallel with the growth of phylogenetic tool programs leads to developing a new systematic and sophisticated method to predict the bioactivities and chemical entities of natural productproducing species using phylogenetic tools. This study's main objective was to explore the phylogenetic approach's usage to generate the information for future bioprospecting to find antimalarial plant-based drugs. ITS region was used to construct a phylogenetic tree because of the capability to distinguish at the lower level of taxonomic order (between species), and also, a large amount of data has been available in the GenBank. Additionally, this region is commonly used for plant DNA barcoding [32]-[35].

The 280 plant taxa were analyzed and revealed that the medicinal plant used to treat malaria, fever, and tuberculosis was clumped in certain phylogenetic tree lineages (Fig 2). The addition of plants used for tuberculosis treatment was purposed to investigate the clumping pattern in more extraordinary samples, which might alter the clustered pattern. Tuberculosis disease was chosen due to different indication given in compared to malaria symptoms hence may not overlap similar usage of the plants, which could generate bias. Fever is one indication of malaria disease. Furthermore, the study showed that numerous similar plant taxa had been used to treat fever and malaria interchangeably. The tremendous amount of medical treatment categories also will narrow down the target plant selection. However, it could 
generate a more scattered pattern rather than the result of a single medical condition category.

Our finding revealed that plants used for malaria treatment were clumped in several plants, including Rubiaceae, Apocynaceae, Euphorbiaceae, and clumped in Asteraceae. Plants used for fever also showed to be clumped in Asteraceae and Cucurbitaceae. On the other hand, plants used for tuberculosis were showed quite scattered in several plants' families but showed to be clumped in the Apiaceae family (Fig 2). The clumping occurrence found an interesting finding on the plant taxa related to antimalarial drugs producing species, the Artemisia annua and Cinchona officinalis producing artemisinin and quinine, respectively. However, clustered occurrence on related plant taxa with $C$. officinalis was showed a relatively small clumping. It may be happened because of the small amount of the plant taxa been used for malaria treatment from family Rubiaceae (8 taxa, Fig 3). On the other hand, in Asteraceae (family of A. annua), it showed a greater clumping pattern and covered both malaria and fever treatment. This was supported from the analysis of the number of our medicinal plant's database; Asteraceae was shown to be the most frequent medicinal plants used for malaria and fever by the people (Fig 3).

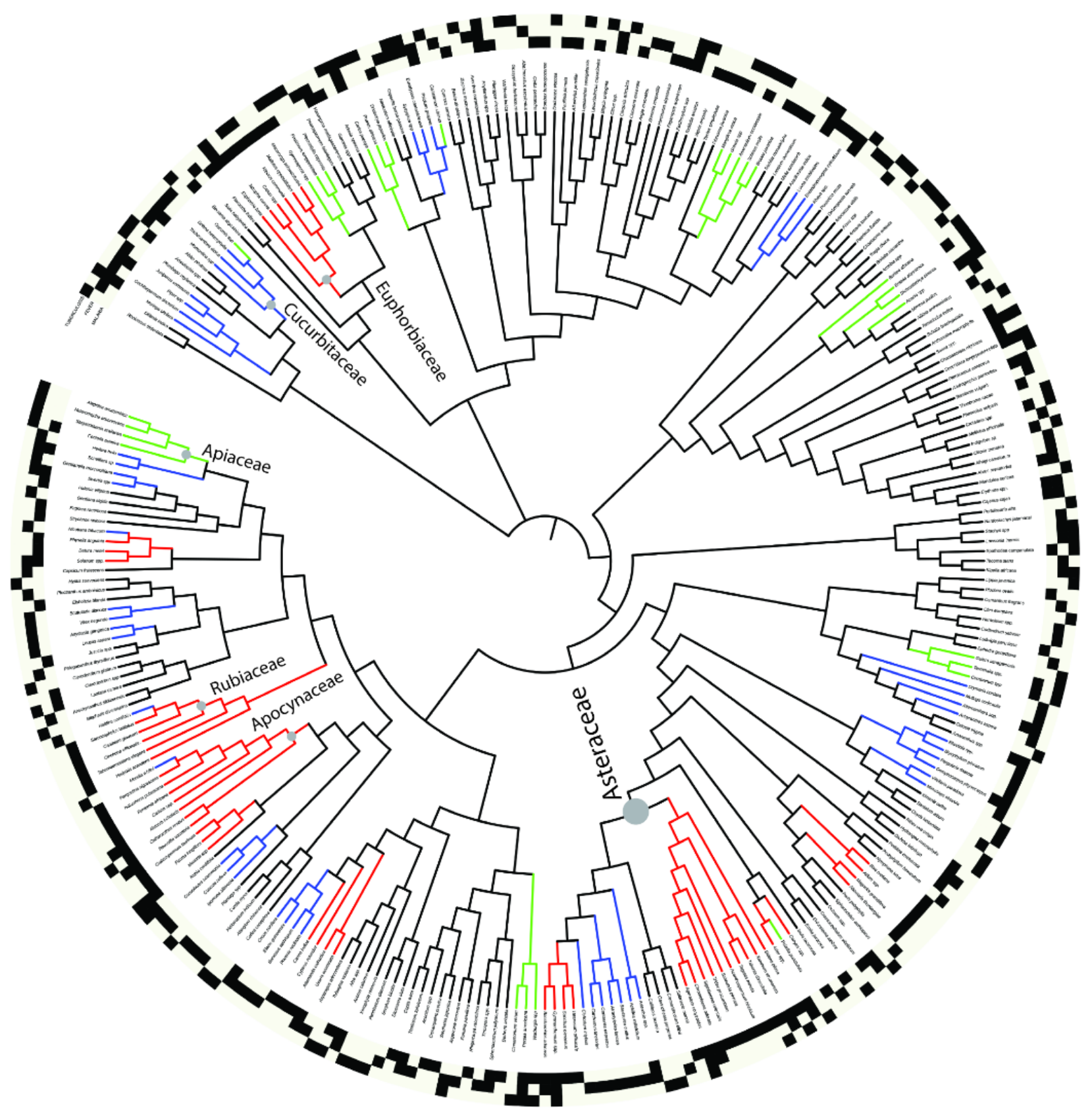

Fig. 2 Phylogenetic mapping of 280 medicinal plants taxa used by people to treat malaria (red branch), fever (blue branch) and tuberculosis (green branch) 

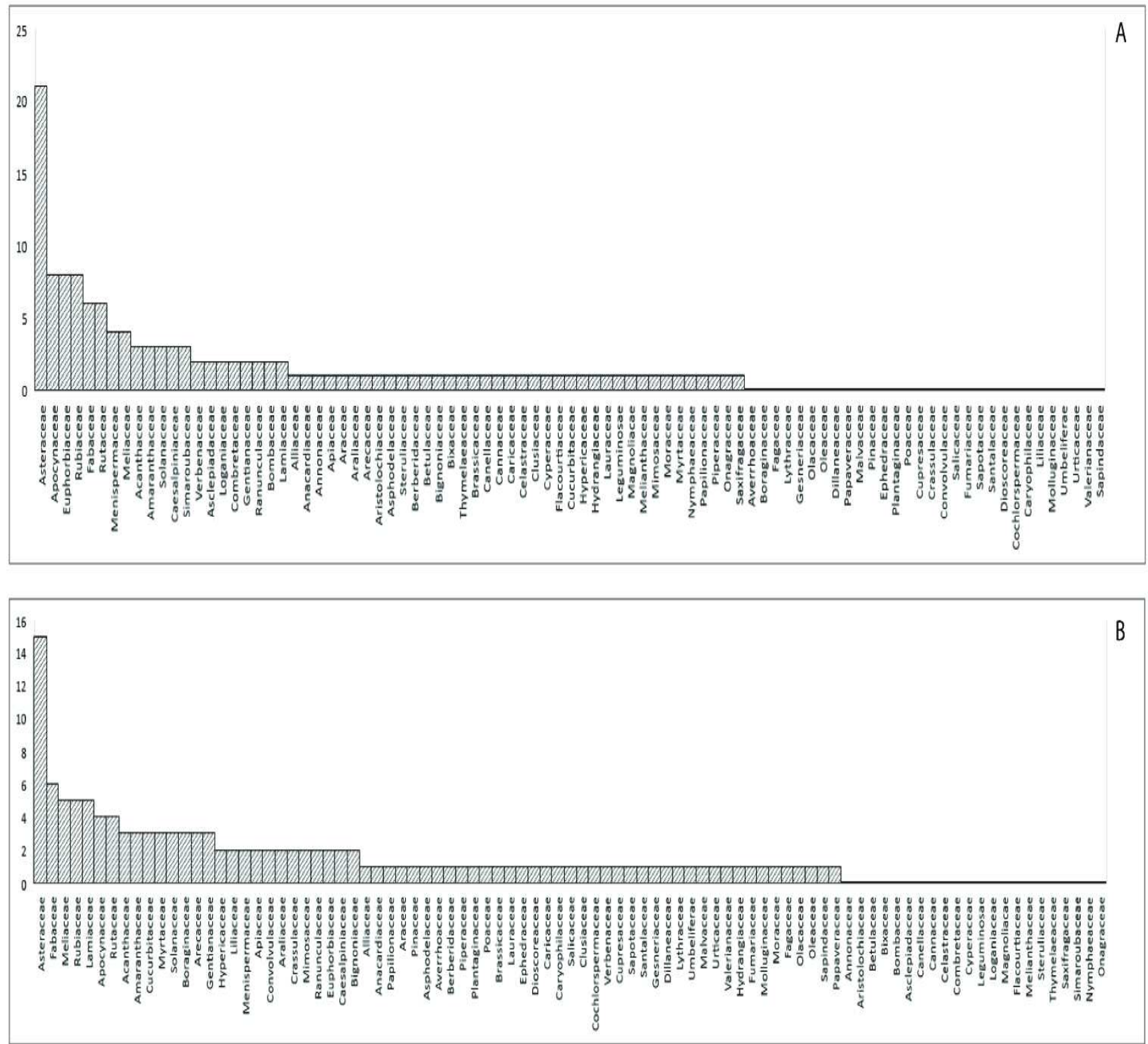

Fig. 3 Frequency of medicinal plant's families used by people to treat malaria (A) and fever (B)

Further investigation by extracting malaria and fever (tuberculosis was excluded) showed a quite similar pattern. A total of 221 plant taxa from 90 medicinal plants used for malaria, 84 for fever, and 47 plants taxa for both conditions were further analyzed to construct the phylogenetic tree. A quite similar pattern as shown in figure 2, antimalarial medicinal plants were also clustered in Apocynaceae, Rubiaceae, and Euphorbiaceae. However, additional clumping patterns were shown in Rutaceae and Fabaceae families. Furthermore, the clustered pattern still remains the same, which was greatly clumped in the Asteraceae family and covered the fever medical purpose condition (Fig 4).

The Asteraceae family showed to be the major source of plants to get the therapeutic properties to treat both malaria and fever by the people across indigenous communities.
Besides Asteraceae, people also frequently use the plant species belong to Apocynaceae, Euphorbiaceae, Rubiaceae, Fabaceae, and Rutaceae to treat malaria (Fig 3). This frequency analysis supported clumping patterns in the constructed phylogenetic tree (Fig 4.). On the other hand, Fabaceae, Meliaceae, Rubiaceae, and Lamiaceae, which are often used for fever treatment, did not show the clustered signal on the generated phylogenetic tree (Fig $3 \& 4$ ). Only the Asteraceae family showed to be clustered in the fever medical treatment category. This result suggested that comparing to the other families, a strong clustered signal for malaria and fever as its associated symptom has occurred to show in the Asteraceae family. Hence, Asteraceae is needed to be explored further. 


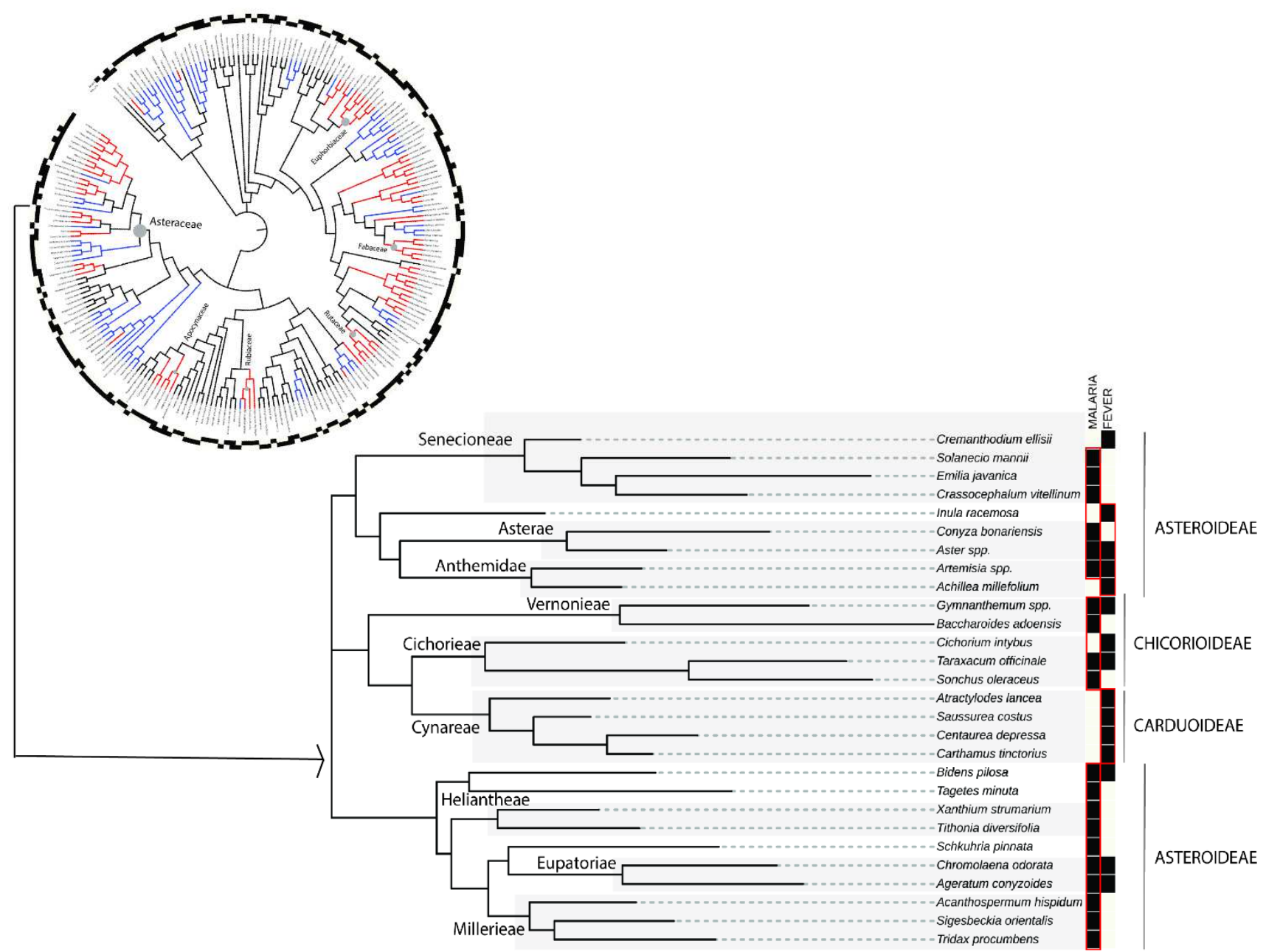

Fig. 4 Phylogenetic mapping of 221 medicinal plants used by the people from various indigenous culture to treat malaria and fever. Red branched represent the plant used for malarial treatment while blue branched for fever treatment.

Classification of the sub-family and tribes was done based on Panero and Funk 2002, with Feddeeae [36]. Medicinal plants taxa for malaria treatment were shown to be clumped in the Asteraceae family. These plants were known as a taxa member belongs to 2 sub-family of Asteraceae, Asteroidea, and Chicorioideae. On the other hand, the plants used for fever clumped in sub-family Carduoideae (tribe Cynareae: Atractylodeslancea, Saussureacoctus, Centaurea depressa, andCarthamustinctorius).

As shown in fig 4, the plants belong to the sub-family Asteroideae were from various tribe, including Senecioeae (Cremanthodiumellisii, Solaneciomannii, Emilia javanica, and Crassocephalumvitellinum), Inuleae (Inularacemosa), Asterae (Conyzabonariensis and Aster spp.), Anthemidae (Artemisia spp. and Achillea millefolium) and Heliantheae alliance. In Heliantheae alliance (Helianthodaesupertribe), the medicinal plants which belonged to Coreopsideae (Bidenspilosa), Tagetae (Tagetesminuta), Heliantheae (Xanthium strumarium and Tithoniadiversifolia), Eupatoriae (Chromolaenaodorata and Ageratumconyzoides), and Millerieae (Acanthospermumhispidium, Sigesbeckiaorientalis, and Tridaxprocumbens), were shown to have strong clustered clumping pattern for malaria category. On the other hand, medicinal plants belong to sub- family Cichorideaewhich were categorized into 2 tribes,Vernonieae (Gymnanthemum spp Baccharoidesadonensis) and Cichorieae (Cichoriumintybus, Taraxacum officinalis and Sonchusoleraceous), also showed the clustered pattern in malaria medical category.

As mentioned before, a strong clumping pattern was occurred in Heliantheae alliance (Fig 4). Furthermore, based on the review of the scientific evidence, almost plants clustered in this group showed to have antiplasmodial activity resulted from laboratory testing. Ethyl acetate extract and 12 fractions of $B$. pilosa was reported have in vivo antiplasmodial activity [37]. Isolated compounds from $X$. stumarium showed excellent in vitro antimalarial activity against 3D7 P. Falciparum [38]. T. diversifolia showed antimalarial activity [39]. Leaves fractions of $C$. Odorata also showed potent activity during in vivo and in vitro study against chloroquine-sensitive and chloroquine-resistant $P$. Falciparum [40]. On the other hand, A. Conyzoides acted good antimalarial activity via in vivo study [41]. The methanolic extract of $\mathrm{A}$. hispidumwas reported moderate inhibitory activity against Dd2 and 3D7 P. falciparumas well [42]. At last, water and ethanolic extract of $T$. procumbens was reported to act as antiplasmodial against chloroquineresistant $P$. Falciparum [43]. Eventually, this finding 
suggested that the clumping pattern of traditional uses of the plants as an antimalarial is in line with the generated result from bioactivity testing in the laboratory. Hence, the clustered signal is worth using as a baseline for candidate plant selection for further experimental study.

Asteraceae is the largest family which has been divided into 12 sub-families [44], [45]. This group member synthesizes the terpenoids, flavonoids, and polyacetylenes, while the sesquiterpene lactones are the compound group used as its taxonomic markers[46]. Asteroidea, known to be the largest subfamily in Asteraceaewhich is comprised of approximately 15,500 species belong to 1,229 genera and 20 tribes (represent $60-70 \%$ in the family) [36], [47]. The important antimalarial drug-producing species (the A. annua) belong to this group; hence, this could be indicated that related species of this plant could have similar antimalarial bioactivities due to chemical properties relation. This suggestion is based on the research conducted on the plant member of Amaryllidaceae, which showed the significant correlation between the phylogeny, chemical diversity, and their bioactivity. The central nervous system (CNS) was investigated related to activities, such as inhibition of acetylcholinesterase and binding activity to the serotonin reuptake transporter with the alkaloid diversity and correlated significantly with the phylogeny of taxa tested [48]. Accordingly, our finding could be used as a baseline to select the candidate plant for antimalaria. Using the phylogeny approach, we can directly "hit" the hot group, which showed the clumping pattern for further investigation, thus minimizing the expenditure and time of the research. Additionally, it may be used as a predictor for the responsible bioactive compound shared between related species.

The chemical class occurrence in tribes of Asteraceae has been conducted statistically using 25,392 samples from 11 chemical classes of compound [49]. The chemical classes are monoterpenes, sesquiterpenes, sesquiterpene lactones, diterpenes, triterpenes, polyacetylenes, coumarines, benzofurans, acetophenones, and phenyl propane. The showed that that the occurrence of the prevalent chemical in each tribe were different. In Asterae tribe, diterpenes (46.7\%) is the most occurred compounds, followed by flavonoids $(20.7 \%)$, coumarines $(6.9 \%)$, sesquiterpenoids (5.9\%), polyacetylenes $(4.5 \%)$, acetophenones $(3.5 \%)$, triterpenes $(3.3 \%)$, phenyl-propane $(3.1 \%)$, monoterpenes $(2.8 \%)$, benzofurans $(2.1 \%)$, and the rarest is sesquiterpene lactones (0,5\%). In Anthemidae tribe, the most occurred compounds are monoterpenes $(59.2 \%)$, followed by flavonoids $(14.2 \%)$, sesquiterpene lactones (13.7\%), sesquiterpenoids (5\%), polyacetylenes $(4.1 \%)$, coumarines $(2.8 \%)$, triterpenes $(0.5 \%)$, acetophenones $(0.4 \%)$, and diterpenes $(0.1 \%)$, respectively. Benzofuranes and phenyl-propane is found to be not have occurred in this tribe[50]. STLs santanolides, germacranolides, and guaianolides are known to be present in this tribe, and all of them present in Artemisia genus[49]. In Senecioneae tribe, sesquiterpenoids $(61.7 \%)$ is the most occurred chemicals followed by monoterpenes (7.9\%), acetophenones $(6.6 \%)$, sesquiterpene lactones $(5.8 \%)$, benzofuranes $(4.7 \%)$, diterpenes $(3.6 \%)$, polyacetylenes (3.5\%), phenyl-propanes $(2.1 \%)$, flavonoids $(1.1 \%)$, and coumarines (0.2\%)[50]. Typical STLs which is being the character of this tribe, are eremophilanolides [49]. In
Heliantheae tribe, sesquiterpene lactones (27.6\%) are most prevalent chemicals, followed by diterpenes $(22.5 \%)$, flavonoids (18.1\%), monoterpenes $(10.1 \%)$, acetophenones $(5.5 \%)$, sesquiterpene lactones $(4.6 \%)$, polyacetylenes (4.6\%), phenyl-propane $(2.7 \%)$, benzofuran $(2.4 \%)$, triterpenes $(1.4 \%)$, and coumarines $(0.5 \%)$, respectively. In Eupatorieae tribe, diterpenes $(24.1 \%)$ is most prevalent compound followed by sesquiterpene lactones (17.1\%), monoterpenes $(14.6 \%)$, acetophenones $(12.9 \%)$, flavonoids $(11.4 \%)$, sesquiterpenoids (7\%), benzofuran (6.8\%), triterpenes $(3.4 \%)$, coumarin $(1.2 \%)$, phenyl-propane $(1.2 \%)$ and polyacetylenes $(0.4 \%)$. In Inuleae tribe, the most prevalent chemicals aresesquiterpenoids $(29.0 \%)$, sesquiterpene lactones (21.8\%), monoterpenes (16.2\%), diterpenes $(14.8 \%)$, flavonoids $(5.9 \%)$, acetophenones $(3.0 \%)$, polyacetylenes $(2.4 \%)$, phenyl propane $(2.2 \%)$, triterpenes $(1.8 \%)$, benzofuran $(1.7 \%)$ and coumarin $(1.2 \%)$, respectively. In Vernonieae tribe, the most prevalent chemicals are sesquiterpene lactones $(59.6 \%)$, triterpenes $(22.5 \%)$, coumarin $(6.0 \%)$, flavonoids $(3.6 \%)$, monoterpenes $(3.1 \%)$, sesquiterpenes $(2.5 \%)$, phenylpropanes $(1.2 \%)$, polyacetylenes $(0.8 \%)$ and diterpenes $(0.5 \%)$, respectively.

On the other hand, benzofuran and acetophenones are not occurred in this tribe. In the Cichoriae tribe, differently from other tribes, Cichorieae is known to be yielded only guaianes, germacrene, and eudesmanes sesquiterpenes. Additionally, guaianolides are the most prevalent in this tribe (76.6\%). The presence of carboxylic acid and sugars residues in the molecules is the structure's common feature [51]. Flavonoids $(56.0 \%)$ are the chemicals classed compounds that were prominent in Cichoriae, followed by sesquiterpene lactones $(14.8 \%)$, sesquiterpenoids $(12.3 \%)$, coumarines $(7.7 \%)$, triterpenes $(3.2 \%)$, phenyl-propane $(2.4 \%)$, and monoterpenes $(2.1 \%)$, respectively. In contrast, benzofuranes and acetophenone, and diterpenes are absent in this tribe[50]. In contrast, a tribe of Cynareae is known to be a promising source of flavonoids and ecdysteroids. Coumarines, triterpenes, and alkaloids are also prevalent in this tribe [52].

A chemometric study using those chemical groups indicated the capability of chemicals data to contribute intrafamily classification in Asteraceae. However, rather than chemical groups, the specific chemical is needed to refine the classification within the tribes [49]. Additionally, another work also showed that trees constructed based on chemical group characters showed different relationship tree of some Asteraceae tribes compared to the trees constructed by morphological (Bremer's classification) and molecular characters (Jansen's classification). Inconsistent and incomplete chemical reports give the challenge of using the chemicals data as taxonomic tools. However, it gives visible information to investigate the adaptiphytochemical's adaptive function Ecological factors that influence the production of phytochemicals and their different speed in evolution compared to morphological characters also may give challenges using those chemicals data.

Nevertheless, sesquiterpene lactones (STLs) are valuable chemical classes as taxonomic markers in the Asteraceae family [54]. Hence, the related species in the same groups of Asteraceae may share similar chemical structure properties of STLs (e.g., Heliantheae and Inuleae shared similar skeletal types, many genera of Vernonieae produce a similar type of 
guaianolides, Inuleae produced typical xanthanolides, and many members of Eupatoriae produced a similar type of germacranolides and guaianolides) [55]. Accordingly, every tribe indicated their typical STLs might be used as a predictor to search the responsible bioactive compounds.

STLs, the C-15 terpenes containing $\alpha$-methylene- $\gamma$-lactone, have high bioactivity due to the presence of their electrophilic moiety (exocyclic enoate), which able to trap the nucleophilic active site of target enzymes [56]-[59]. Artemisinin, the important commercial antimalarial drug, is the STLs which is characterized by its endoperoxide bridge as their known responsible structure in its mechanism of action as an antimalarial drug [60], [61]. STLs may become a major source of the antimalarial drug because they can pass the blood-brain barrier hence may be useful for cerebral malaria therapy. These compounds have potency as antiinflammatory, antioxidant, neuroprotective, and phytotoxic [57], which may lead to adjuvant therapy or apicoplast drug target.

Our finding showed the occurrence of a great clumping pattern in a group of plant related to STLs artemisinin producing species (A. annua), which may indicate the possibility of sharing chemical structure properties resulting in similar malaria healing activity (Fig. 4). Artemisinin is the STLs formed by joined various biosynthetic processes from a common origin, and about 170 types of STLs are found prevalent in several tribes, including Anthemideae, Inuleae, and Senecioneae [50]. This study revealed that the plant members of the Heliantheae alliance showed a strong clumping pattern in the malaria category. However, there was no report about the typical chemicals in this group, requiring further investigation.

Lastly, our finding supported the other previous research that suggest using the phylogeny approach and ethnobotanical bioprospecting for future plant-based drug discovery. The clumping pattern of the plants used to treat similar medical conditions may indicate the possibility of sharing chemical properties. This approach could be used to find substitution alternative taxa to treat certain similar medical conditions when the supply or availability of the medicinal taxa is ecologically threatened [48]. To find the new antimalarial drug candidate, this finding may be used as a baseline for selecting the target plant group rather than making a random selection. The hot node group may be selected for further laboratory testing. However, because of the plant's ecological factors may affect the production of secondary metabolites responsible for its bioactivity, further laboratory testing is needed to investigate this finding.

\section{IV.CONCLUSION}

Ethnobotanical plants used for the treatment of malaria and fever were clumped mostly in Asteraceae family derived from various tribes. The interesting, clustered patterns of plants used for malaria were shown in the Asteroidea sub-family group, related to the antimalarial drug-producing species. Additionally, the tribe Heliantheae alliance showed a strong clumping pattern of a plant used for malaria, while plants used for fever showed clumped in the Carduoideae tribe. This finding may be used as a baseline for selecting the target plant for future bioprospecting to discover new antimalaria. At least, the phylogeny approach may become new interesting tools to predict the bioactivity of medicinal plants.

\section{ACKNOWLEDGMENT}

The authors are grateful to the staff of the College of Public Health Sciences for their assistance. This research is funded by the AEC Scholarship of Chulalongkorn University and the $90^{\text {th }}$ Anniversary of Chulalongkorn University Scholarship, Thailand.

\section{REFERENCES}

[1] S. B. McCreath and R. Delgoda, Pharmacognosy: Fundamentals, applications and strategies. Academic Press, 2017.

[2] M. A. Aziz, A. H. Khan, M. Adnan, and I. Izatullah, "Traditional uses of medicinal plants reported by the indigenous communities and local herbal practitioners of Bajaur Agency, Federally Administrated Tribal Areas, Pakistan," J. Ethnopharmacol., vol. 198, pp. 268-281, 2017, doi: https://doi.org/10.1016/j.jep.2017.01.024.

[3] H. Shaheen, M. F. Qaseem, M. S. Amjad, and P. Bruschi, "Exploration of ethno-medicinal knowledge among rural communities of Pearl Valley; Rawalakot, District Poonch Azad Jammu and Kashmir," PLoS One, vol. 12, no. 9, p. e0183956, 2017.

[4] I. Süntar, "Importance of ethnopharmacological studies in drug discovery: role of medicinal plants," Phytochem. Rev., pp. 1-11, 2019.

[5] S. Wang, G. Dong, and C. Sheng, "Structural simplification: an efficient strategy in lead optimization," Acta Pharm. Sin. B, vol. 9, no. 5, pp. 880-901, 2019, doi: https://doi.org/10.1016/j.apsb.2019.05.004.

[6] S. Krishnaprabu, "Therapeutic potential of medicinal plants: A," J. Pharmacogn. Phytochem., vol. 9, no. 2, pp. 2228-2233, 2020.

[7] S.-L. C. Haris et al., "Phylogenies reveal predictive power of traditional medicine in bioprospecting," J Proc. Natl. Acad. Sci., vol. 109, no. 39, pp. 15835-15840, 2012, doi: https://doi.org/10.1073/pnas.1202242109.

[8] J. B. Calixto, "The role of natural products in modern drug discovery.," An. Acad. Bras. Cienc., vol. 91, 2019.

[9] M. J. D., V. S. K., and H. J. T., "Plant natural products: Back to the future or into extinction," Phytochemistry, vol. 68, no. 14, pp. $2015-$ 2022, 2007, doi: https://doi.org/10.1016/j.phytochem.2007.04.032.

[10] A. Rasool, K. M. Bhat, A. A. Sheikh, A. Jan, and S. Hassan, "Medicinal plants: Role, distribution and future," J. Pharmacogn. Phytochem., vol. 9, no. 2, pp. 2111-2114, 2020.

[11] E. J. Buenz, R. Verpoorte, and B. A. Bauer, "The Ethnopharmacologic Contribution to Bioprospecting Natural Products," Annu. Rev. Pharmacol. Toxicol., vol. 58, no. 1, pp. 509-530, 2018, doi: 10.1146/annurev-pharmtox-010617-052703.

[12] E. Guzman and J. Molina, "The predictive utility of the plant phylogeny in identifying sources of cardiovascular drugs," Pharm. Biol., vol. 56, no. 1, pp. 154-164, 2018, doi: 10.1080/13880209.2018.1444642.

[13] S. Vivekanand and S. I. Neil, "Bioinformatics opportunities for identification and study of medicinal plants," Brief. Bioinform., vol. 14, no. 2, pp. 238-250, 2012, doi: https://doi.org/10.1093/bib/bbs021.

[14] I. Zahoor, A. Shafi, K. M. Fazili, and E. Haq, "Bioinformatics and Medicinal Plant Research: Current Scenario," in Essentials of Bioinformatics, Volume III, Springer, 2019, pp. 141-157.

[15] N. Rummun, V. S. Neergheen-Bhujun, K. B. Pynee, C. Baider, and T. Bahorun, "The role of endemic plants in Mauritian traditional medicine - Potential therapeutic benefits or placebo effect?" $J$. Ethnopharmacol., vol. 213, pp. 111-117, 2018, doi: https://doi.org/10.1016/j.jep.2017.10.006.

[16] C. Beedie et al., "'Caution, this treatment is a placebo. It might work, but it might not': why emerging mechanistic evidence for placebo effects does not legitimise complementary and alternative medicines in sport," Br. J. Sports Med., vol. 52, no. 13, pp. 817 LP-818, Jul. 2018, doi: 10.1136/bjsports-2017-097747.

[17] S. Singh, D. B. Singh, S. Singh, R. Shukla, P. W. Ramteke, and K. Misra, "Exploring medicinal plant legacy for drug discovery in postgenomic era," Proc. Natl. Acad. Sci. India Sect. B Biol. Sci., vol. 89, no. 4, pp. 1141-1151, 2019.

[18] H.-G. Morten, E. Madeleine, R. Nina, D. R. R, and S.-L. C. Haris, "Using evolutionary tools to search for novel psychoactive plants," $J$ Plant Genet. Resour., vol. 14, no. 4, pp. 246-255, 2016, doi: https://doi.org/10.1017/S1479262116000344. 
[19] Y. Kowiyou, D. B. H, and M. A. Muthama, "Phylogenetic exploration of commonly used medicinal plants in South Africa," Mol Ecol Resour, vol. 15, no. 2, pp. 405-413, 2015, doi: https://doi.org/10.1111/1755-0998.12310.

[20] R. Delgoda and J. E. Murray, "Chapter 7 - Evolutionary Perspectives on the Role of Plant Secondary Metabolites," S. Badal and R. B. T.-P. Delgoda, Eds. Boston: Academic Press, 2017, pp. 93-100.

[21] M. Erb and D. J. Kliebenstein, "Plant secondary metabolites as defenses, regulators, and primary metabolites: the blurred functional trichotomy," Plant Physiol., vol. 184, no. 1, pp. 39-52, 2020.

[22] Z. Feng et al., "Clustered patterns of species origins of nature-derived drugs and clues for future bioprospecting," Proc. Natl. Acad. Sci. U. S. A., vol. 108, no. 31, pp. 12943-12948, 2011, doi: https://doi.org/10.1073/pnas.1107336108.

[23] A. N. Aid and M. Jeanmaire, "The ethnobotany of psychoactive plant use: a phylogenetic perspective," PeerJ, vol. 4, pp. e2546-e2546, 2016, doi: https://doi.org/10.7717/peerj.2546.

[24] R. Varo, C. Erice, S. Johnson, Q. Bassat, and K. C. Kain, "Clinical trials to assess adjuvant therapeutics for severe malaria," Malar. J., vol. 19 , no. 1, p. 268 , 2020, doi: 10.1186/s12936-020-03340-3.

[25] O. Manel, A. Jean-Michel, P. Lucie, and B.-V. Françoise, "Plasmodium falciparum resistance to artemisinin-based combination therapies: A sword of Damocles in the path toward malaria elimination," $J$ Parasite, vol. 25, 2018, doi: https://doi.org/10.1051/parasite/2018021.

[26] WHO, World Malaria Report 2019. World Health Organization 2019, 2019.

[27] R. Pasupureddy, S. Seshadri, V. Pande, R. Dixit, and K. C. Pandey, "Current scenario and future strategies to fight artemisinin resistance," Parasitol. Res., vol. 118, no. 1, pp. 29-42, 2019.

[28] E. A. Ashley and A. P. Phyo, "Drugs in Development for Malaria," Drugs, vol. 78, no. 9, pp. 861-879, 2018, doi: 10.1007/s40265-0180911-9.

[29] T. Hanscheid and D. W. Hardisty, "How 'resistant' is artemisinin resistant malaria? - The risks of ambiguity using the term 'resistant' malaria," Travel Med. Infect. Dis., vol. 24, pp. 23-24, 2018, doi: https://doi.org/10.1016/j.tmaid.2018.04.008.

[30] J. Wang, C. Xu, F. L. Liao, T. Jiang, S. Krishna, and Y. Tu, "A temporizing solution to 'artemisinin resistance,"” N. Engl. J. Med., vol. 380 , no. 22 , pp. 2087-2089, 2019

[31] T. Chookajorn, "How to combat emerging artemisinin resistance: Lessons from 'The Three Little Pigs,"” PLoS Pathog., vol. 14, no. 4 , p. e1006923, 2018

[32] K. Yong, D. Zhiyan, Z. Runguo, and L. Wenxing, "DNA barcoding analysis and phylogenetic relationships of tree species in tropical cloud forests," Sci. Rep., vol. 7, no. 1, pp. 1-9, 2017, doi https://doi.org/10.1038/s41598-017-13057-0.

[33] T. Gunnels, M. Creswell, J. McFerrin, and J. B. Whittall, "The ITS region provides a reliable DNA barcode for identifying reishi/lingzhi (Ganoderma) from herbal supplements," PLoS One, vol. 15, no. 11, p. e0236774, 2020.

[34] B. P. Murphy and P. J. Tranel, "Identification and Validation of Amaranthus Species-Specific SNPs within the ITS Region: Applications in Quantitative Species Identification," Crop Sci., vol. 58, no. 1, pp. 304-311, 2018.

[35] L. V Ya-Na et al., "Identification of medicinal plants within the Apocynaceae family using ITS2 and psbA-trnH barcodes," Chin. $J$ Nat. Med., vol. 18, no. 8, pp. 594-605, 2020.

[36] V. Funk, A. Susanna, T. Stuessy, and R. Bayer, Systematics, Evolution, and Biogeography of Compositae. Veinna, Austria: International Association for Plant Taxonomy, Institue of Botany, University of Vienna, 2009.

[37] N. A. C. Nadia et al., "Antimalarial Activity of Ethyl Acetate Extract and Fraction of Bidens pilosa against Plasmodium berghei (ANKA)," J. Parasitol. Res., vol. 2020, 2020

[38] R. K. Sahoo et al., "Phytochemical constituents from Xanthium strumarium L. and evaluation of their in vitro antimalarial activities," South African J. Bot., vol. 135, pp. 35-40, 2020, doi: https://doi.org/10.1016/j.sajb.2020.08.006.

[39] A. A. Ajao and A. N. Moteetee, "Tithonia diversifolia (Hemsl) A. Gray. (Asteraceae: Heliantheae), an invasive plant of significant ethnopharmacological importance: A review," South African J. Bot., vol. 113, pp. 396-403, 2017, doi: https://doi.org/10.1016/j.sajb.2017.09.017.

[40] I. C. Ezenyi, O. A. Salawu, R. Kulkarni, and M. Emeje, "Antiplasmodial activity-aided isolation and identification of quercetin-4'-methyl ether in Chromolaena odorata leaf fraction with high activity against chloroquine-resistant Plasmodium falciparum," $J$ Parasitol. Res., vol. 113, no. 12 LB-Ezenyi2014, pp. 4415-4422, 2014, doi: 10.1007/s00436-014-4119-y.

[41] I. H. Ifijen, M. Maliki, O. K. Ogbeide, R. O. Okonkwo, S. O. Omorogbe, and E. U. Ikhuoria, "Chemical Substances and in-Vivo Antiplasmodial Activity of Ageratum Conyzoides in Plasmodium Berghei Infected Mice," J. Appl. Sci. Environ. Manag., vol. 23, no. 10, pp. 1813-1817, 2019.

[42] F. Koukouikila-Koussounda et al., "In vitro evaluation of antiplasmodial activity of extracts of Acanthospermum hispidum DC (Asteraceae) and Ficus thonningii blume (Moraceae), two plants used in traditional medicine in the Republic of Congo," African J. Tradit. Complement. Altern. Med., vol. 10, no. 2, pp. 270-276, 2013.

[43] P. Ghosh, S. Biswas, M. Biswas, A. Dutta, S. Sil, and S. Chatterjee, "Morphological, Ethno biological and Phytopharmacological Attributes of Tridax procumbensLinn.(Asteraceae): A Review," Int. J. Sci. Res. Biol. Sci. Vol, vol. 6, p. 2, 2019.

[44] C. McDonald-Spicer, N. J. Knerr, F. Encinas-Viso, and A. N. SchmidtLebuhn, "Big data for a large clade: Bioregionalization and ancestral range estimation in the daisy family (Asteraceae)," J. Biogeogr., vol. 46, no. 2, pp. 255-267, 2019.

[45] J. R. Mandel et al., "The Compositae tree of life in the age of phylogenomics," J. Syst. Evol., vol. 55, no. 4, pp. 405-410, 2017.

[46] M. E. Gallon, M. Monge, R. Casoti, F. B. Da Costa, J. Semir, and L. Gobbo-Neto, "Metabolomic analysis applied to chemosystematics and evolution of megadiverse Brazilian Vernonieae (Asteraceae)," Phytochemistry, vol. 150, pp. 93-105, 2018.

[47] S. V. Patricia and M. V. Susana, Sesquiterpene Lactones: Advances in Their Chemistry and Biological Aspects. Springer, 2018.

[48] R. Nina et al., "Can phylogeny predict chemical diversity and potential medicinal activity of plants? A case study of Amaryllidaceae," BMC Evol. Biol., vol. 12, no. 1, p. 182, 2012, doi: https://doi.org/10.1186/1471-2148-12-182.

[49] A. SAV, F. MJP, E. VP, and C.-B. D, "Chemosystematic studies of natural compounds isolated from Asteraceae: characterization of tribes by principal component analysis," Chemom. Intell. Lab. Syst., vol. 56, no. 1, pp. 27-37, 2001, doi: https://doi.org/10.1016/S01697439(01)00103-4.

[50] S. S., The Chemistry of Natural Products: 6: Plenary Lectures Presented at the Sixth International Symposium on the Chemistry of Natural Products. Elsevier Science, 2013.

[51] Z. Christian, "Sesquiterpene lactones and their precursors as chemosystematic markers in the tribe Cichorieae of the Asteraceae," Phytochemistry, vol. 69, no. 12, pp. 2270-2296, 2008, doi: https://doi.org/10.1016/j.phytochem.2008.06.013.

[52] K. E., Z. L., and R. A., "Secondary metabolites of some Siberian species of plants tribe Cynareae (Asteraceae)," South African J. Bot., vol. $125, \quad$ pp. 24-29, 2019, doi: https://doi.org/10.1016/j.sajb.2019.06.022.

[53] C. Lalita, E. Vicente, F. Marcelo, S. Marcus, and M. Tom, "A Phylogenetic Analysis of Tribes of the Asteraceae Based on Phytochemical Data," Nat. Prod. Commun., vol. 2, pp. 277-285, 2007

[54] A. M. Feitosa, S. Luciana, D. C. F. Batista, and S. M. Tullius, "Chemotaxonomic Study of Sesquiterpene Lactones of Asteraceae: Classical and Modern Methods," in Sesquiterpene Lactones: Advances in their Chemistry and Biological Aspects, V. P. Sülsen and V. S. Martino, Eds. Cham: Springer International Publishing, 2018, pp. 3145 .

[55] S. F. C., "Sesquiterpene lactones as taxonomic characters in the asteraceae," Bot. Rev., vol. 48, no. 2, pp. 121-594, 1982, doi: http://dx.doi.org/10.1007/BF02919191.

[56] B. Joshi, S. Hendrickx, L. B. Magar, N. Parajuli, P. Dorny, and L. Maes, "In vitro antileishmanial and antimalarial activity of selected plants of Nepal," J Intercult Ethnopharmacol, vol. 5, no. 4, pp. 383389, 2016, doi: 10.5455/jice.20160728031236.

[57] M. Laila, C. Oliver, S. Pedro, S. Farukh, and S. A. ML, "Applications of Sesquiterpene Lactones: A Review of Some Potential Success Cases," J Appl. Sci., vol. 10, no. 9, p. 3001, 2020, doi: http://10.3390/app10093001.

[58] S. K. Mandal et al. "Natural Sesquiterpene Lactones in the Prevention and Treatment of Inflammatory Disorders and cancer: A Systematic Study of this Emerging Therapeutic Approach based on Chemical and Pharmacological Aspect," Lett. Drug Des. Discov., vol. 17, no. 9, pp. $1102-1116,2020$

[59] T. J. Schmidt, "Structure-Activity and Activity-Activity Relationships of Sesquiterpene Lactones BT - Sesquiterpene Lactones: Advances in their Chemistry and Biological Aspects," V. P. Sülsen and V. S. 
Martino, Eds. Cham: Springer International Publishing, 2018, pp. 349-371.

[60] O. P. M., B. V. E., and W. S. A., "The molecular mechanism of action of artemisinin--the debate continues," Molecules, vol. 15, no. 3, pp.
1705-1721, 2010, doi: http://10.3390/molecules15031705.

[61] S. Gunjan et al., "Artemisinin derivatives and synthetic trioxane trigger apoptotic cell death in asexual stages of plasmodium," Front. Cell. Infect. Microbiol., vol. 8, p. 256, 2018. 